\title{
T rastorno de evitación y restricción de la ingesta de alimentos (ARFID) con síntomas obsesivos en un niño con Trastorno del Espectro Autista, a propósito de un caso clínico
}

\author{
Avoidance and restrction of food intake disorder (ARFID) associated \\ with obsessive symptoms ina child with autism spectrum disorder \\ on the subject of a clinical case
}

\author{
María A. Huete-Córdova ${ }^{1,2, a}$, Manuel D. Arce-Martino ${ }^{1,2, b}$
}

Resumen

Se presenta el caso de un niño de 11 años que ingresa a la emergencia de pediatría por deshidratación severa, dolor abdominal, vómitos, baja de peso, ideas obsesivas relacionadas a la ingesta de la comida por el temor a vomitar, preocupación obsesiva con relación al consumo de algunos alimentos ricos en carbohidratos por el temor a presentar molestias digestivas, niega miedo a engordar. Durante la hospitalización por la desnutrición proteico calórica severa se descarta otras causas no psiquiátricas y a la evaluación por psiquiatría del niño y del adolescente se obtiene datos característicos del trastorno de espectro autista y trastorno de evitación y restricción de la ingesta de alimentos clínica que lo presentaba mucho tiempo anterior, sintomatología que pasó desapercibido a pesar de la disminución del peso corporal, presentando sintomatología de trastorno obsesivo compulsivo (TOC) con predominio de pensamientos obsesivos lo cual agravó el diagnóstico previo. Por lo que se inicia tratamiento farmacológico, psicoterapia individual y familiar obteniendo resultados positivos en el paciente. Por la alta prevalencia de comorbilidades del trastorno del espectro autista, como el caso que presentamos TOC y TERIA, es de importancia su identificación y tratamiento farmacológico y psicoterapéutico para mejor pronóstico.

Palabras clave: Trastorno del Espectro Autista (TEA), Trastorno de Evitación y Restricción de la Ingesta de Alimentos (TERIA) y Trastorno Obsesivo Compulsivo (TOC).

\section{Abstract}

The case of an 11 years old who is admitted to the pediatric emergency room due to severe dehydration, abdominal pain, vomiting, weight loss, obsessive ideas related to food intake due to fear of vomiting, obsessive concern in relation to food is presented. consumption of some foods rich in carbohydrates for fear of digestive disconfort, denies fear of gaining weight. During hospitalization for severe protein-calorie malnutrition, other non-psychiatric causes are ruled out and the psychiatric evaluation of the child and adolescent obtains characteristic data of autism spectrum disorder and clinical avoidance and restriction of food intake disorder that presented it a lot Previous time, symptoms that went unnoticed despite the decrease in body weight, presenting symptoms of obsessive compulsive disorder (OCD) with a predominance of obsessive thoughts, which aggravated the previous diagnosis. Therefore, pharmacotherapy and individual and family psychotherapy is started, obtaining positive results in the patient. Due to the high prevalence of comorbidities of autism spectrum disorder, such as the case that we present with $O C D$ and TERIA, its identification and pharmacological and psychotherapeutic treatment are important for a better prognosis.

Keywords: Autism Spectrum Disorder (ASD), Avoidance and Restriction of Food Intake Disorder (ARFID), Obsessive Compulsive Disorder (OCD).

\footnotetext{
${ }^{I}$ Servicio de Psiquiatría del Niño y del Adolescente, Hospital Nacional Guillermo Almenara Irigoyen. Lima, Perú. ${ }^{2}$ Departamento de Psiquiatría, Hospital Nacional Guillermo Almenara Irigoyen. Lima, Perú. ${ }^{a}$ Médico Psiquiatra, Médico residente de Psiquiatría del Niño $y$ del Adolescente de la Universidad Ricardo Palma (URP). ${ }^{b}$ Psiquiatra de niños y adolescentes.
}

ORCID: Maria Antonieta Huete-Cordova: https://orcid.org/0000-0002-2569-6257

Manuel David Arce-Martino: https://orcid.org/0000-0003-0283-6162 


\section{Introducción}

La principal característica diagnóstica del trastorno de evitación/restricción de la ingesta de alimentos (TERIA) es la evitación o restricción de la ingesta de los alimentos asociada a pérdida de peso significativa, deficiencias nutritivas significativas, dependencia de la nutrición enteral o suplementos por vía oral e interferencia importante en el funcionamiento psicosocial. La ingesta insuficiente o falta de interés se presenta durante la lactancia o primera infancia. TERIA que se basa en las características organolépticas de los alimentos tiende a presentarse en la primera década de vida y la relacionada con consecuencias aversivas puede presentarse a cualquier edad. TERIA comórbido con TEA es más frecuente en varones ${ }^{(1)}$. Los trastornos de la alimentación tienen una prevalencia entre el 5$25 \%$ en niños y jóvenes y de estos el $12-14 \%$ corresponden a pacientes con TERIA, disminuyendo la sintomatología a mayor edad, encontrándose en adolescentes de 15 años a más una prevalencia del $0.3 \%$. Entre las comorbilidades asociadas al TERIA es el TEA, discapacidad intelectual y el trastorno de déficit de atención con hiperactividad, trastorno obsesivo compulsivo y trastornos de ansiedad ${ }^{(2)}$.

El paciente que presentamos desde la infancia presenta sintomatología de TERIA, lo que genera una baja de peso significativa por la ingesta insuficiente y selección de algunos alimentos lo cual está asociado a la clínica del trastorno del espectro autista. La selectividad alimentaria es frecuente en los pacientes con TEA, pudiendo ser el primer síntoma que se manifiesta en niños con trastornos del neurodesarrollo, encontrándose en algunos estudios una prevalencia del 49-89\% de los niños con TEA con hábitos de alimentación atípicos ${ }^{(3)}$.

En el Perú, 15,625 personas padecen el Trastorno del Espectro Autista (TEA) y de esta cifra el $90.6 \%$ corresponde a menores de 11 años ${ }^{(4)}$. El trastorno del espectro autista (TEA) se caracteriza por las principales alteraciones tanto en la comunicación social y conductas repetitivas, mostrando un interés particular por algunas actividades específicas, resistiéndose a los cambios no respondiendo al entorno social. Tiene una prevalencia de 1\% en Estados Unidos de Norteamérica. Con una frecuencia de cuatro a cinco veces más en el sexo masculino en comparación con el femenino. El Trastorno obsesivo compulsivo (TOC) es frecuente en niños y adolescentes, con una prevalencia del $0,5 \%{ }^{(5)}$. El autismo coexiste a altas tasas en pacientes con TOC. Con relación a la comorbilidad en el TEA, aproximadamente el 70\% de los pacientes con TEA pueden tener un trastorno mental, y el $40 \%$ dos o más trastornos mentales. El trastorno de conducta alimentaria de la ingesta de alimentos es una forma característica de presentación del $\mathrm{TEA}^{(6,1)}$. Es frecuente que tengan una dieta nutricionalmente inadecuada, cuya intervención es interdisciplinario ${ }^{(7)}$.

Martin et al. (2020) describieron en su estudio retrospectivo de cohorte abierto, cuyos datos de los pacientes se tomaron de las historias clínicas electrónicas de pacientes de $4 \mathrm{a}$ 17 años, en el sur de Londres y Maudsley NHS Foundation Trust entre 2007 y 2016. Con una muestra de 7922 adolescentes, de los cuales se obtuvo como resultados, 335 tenían diagnóstico de TOC y TEA, 6577 con TOC y 1010 con TEA. Los pacientes con TOC y TEA tendían a ser más jóvenes en el momento del diagnóstico en comparación con el TOC, con mayor frecuencia en varones y eran más propensos a tener una discapacidad intelectual y a tener una comorbilidad. Teniendo como hallazgo que una cuarta parte de los jóvenes con TOC también tenía diagnóstico de TEA, sugiriendo que el TOC está infradiagnosticado en las personas con TEA en la práctica clínica $^{(8)}$. El protocolo de la terapia cognitivo conductual adaptado se asoció con reducciones significativas en los síntomas del TOC, pudiendo ser un tratamiento parcialmente eficaz para pacientes con TOC y TEA ${ }^{(9)}$. Por la alta prevalencia de comorbilidades del trastorno del espectro autista, como el caso que presentamos en un paciente con TEA, TERIA y TOC, es de importancia su identificación sintomatológica, por lo que realizamos una revisión de los diagnósticos y su asociación entre estos trastornos como comorbilidades del TEA, por la importancia del inicio del tratamiento farmacológico y psicoterapéutico para su mejor pronóstico.

\section{Descripción del Caso}

Presentamos el caso de un paciente varón de 11 años, con diagnóstico de Síndrome de Asperger y TERIA que ingresó por la emergencia por síntomas digestivos y desnutrición proteico-calórica severa, presentando sintomatología de TOC lo cual agravó el diagnóstico previo. Los primeros años de vida quedó a cuidado de abuelos maternos, cursa el $6^{\circ}$ grado de primaria, buen rendimiento escolar, sobresaliente. Vive con la madre y los abuelos maternos, madre de 47 años, con hipotiroidismo e hipertensión arterial. El padre tiene 47 años, aparentemente sano. Tío paterno: leucemia. Abuela materna: depresión. Primo materno: TDAH. Padres separados, Tiene como antecedentes personales: es el primer hijo, controles prenatales completos, presentó preeclampsia severa. Parto Cesárea, prematuro EG:36 semanas. Peso:2,600. APGAR:9-9. Lactancia mixta. Desarrollo psicomotor CC:2-3 meses, sedestación:7-8 meses, Camina 18 meses. Primeras palabras 14 meses, estructura oraciones a los 2 años. Caminaba de puntitas, 
giraba alrededor, presentó aleteo de manos. Hiperreactividad a los estímulos sensoriales como sonidos, texturas, olfato. Hiperlaxitud, hipotonía. Recibió terapia sensorial, terapia ocupacional, terapia de lenguaje y rehabilitación por 3 años en Clínica San Juan de Dios. Vacunas completas. Presentaba dificultad en tocar diferentes texturas, no cogía temperas, plastilinas, arena, sintomatología que mejoró, aún persistiendo el no tolerar las costuras de las ropas, las etiquetas, y la correa, por lo que utiliza pantalones con elástico. Comportamiento con los alimentos: Se llenaba de gases, se le tenía que insistir para comer desde pequeño, se demoraba una hora a más tiempo. Separaba los alimentos, no podían estar mezclado, se tornaba irritable o reclamaba si se le junta la comida, seleccionaba una fruta para llevar al colegio por toda una semana, luego recién pedía otra fruta para llevar. Solo aceptaba frutas y carnes picadas. Le gustaba comer con su mano, y las comidas tibio a frío; posteriormente mejoró sintomatología, en la actualidad aún tiene rechazo por los olores fuertes en las comidas y ante el evento de vómito inició nuevamente con el rechazo de alimentos como tallarines y otros, solo come arroz, pollo y ensalada de verduras todos los días en el almuerzo. Con relación al peso, la madre refiere siempre ha sido de contextura delgada.

Psico estresores: Durante la pandemia, no ha salido de casa, se torna temeroso por el temor al contagio de él y su mamá, tornándose ansioso por lo que la madre es obstetra y va al hospital. En clases virtuales prefiere apagar la cámara y no interactúa con compañeros, solo participa cuando lo invitan a dar su respuesta o comentario, tiene buen rendimiento escolar, ocupa los primeros puestos. Le operaron de apendicetomía, primera vez que lo hospitalizaron, tornándose ansioso, sintomatología que disminuyó al explicársele sobre la intervención quirúrgica. Infección por COVID-19.

Motivo de ingreso a la emergencia: Hace 8 meses, al comer regular cantidad de yogurt y cereal, sintió malestar gástrico y vómitos, se asustó mucho cuando empezó a salir la comida por la nariz, decía que se ahogaba, que no podía respirar, se tornó muy ansioso, demoró dos horas aproximadamente para tranquilizarse, toda la noche pensaba que le podía volver a pasar, al persistir los síntomas gástricos tenía miedo de comer, se tornaba ansioso durante las horas de comida, presentaba temblor del cuerpo al pensar que podía vomitar. Hace dos meses presenta dolor abdominal tipo cólico relacionado a la ingesta de comidas con harinas, flatos, náuseas, insomnio, disminuyó la ingesta de alimentos. Hace dos semanas es más persistente el dolor abdominal, se agrega náuseas, no tolerando sólidos ni líquidos, presenta llenura precoz, médico particular le indicó bismutol, ranitidina y metoclopramida. Madre refirió un peso habitual de 30 kilos, llegó a la emergencia con 26 kilos. Ingresando con diagnósticos: Síndrome doloroso abdominal y Deshidratación moderada-severa, síndrome consuntivo infecciosa vs oncológica.

$\mathrm{Al}$ examen físico al ingreso: Paciente despierto poco activo, caquéctico. Peso $26 \mathrm{Kg}$, Talla 141.5 IMC de 13.1 Piel pálida, ojos hundidos, mucosas secas, lengua saburral, no adenopatías, tejido celular subcutáneo disminuido, murmullo vesicular pasa disminuido en hemitórax derecho. No ruidos agregados, ruidos cardíacos rítmicos de buena intensidad no soplos, abdomen no distendido, dolor a la palpación en abdomen superior, se palpa hígado $+-3 \mathrm{~cm}$ debajo del reborde costal derecho, hipoactivo, reflejos osteotendinosos $(+)$ fuerza muscular disminuida. Psicopatológico: Paciente varón aparenta edad cronológica, caquéctico, No establece contacto visual. Colabora parcialmente con la entrevista, tornándose ansioso por la sonda nasogástrica. Despierto, orientado en persona, espacio y tiempo. Niega pseudopercepciones. No conducta alucinatoria. Pensamiento de curso conservado, coherente, concreto, Ideas obsesivas relacionadas a la ingesta de la comida por el temor a vomitar "voy a ahogarme, me va faltar aire voy a vomitar", preocupación obsesiva con relación al consumo de algunos alimentos ricos en carbohidratos "me puede producir gases y náuseas", niega miedo a engordar. Lenguaje fluido de volumen bajo. Ánimo ansioso, poca resonancia afectiva. Capacidad de abstracción disminuida. Hiporexia, restricción de ingesta de carbohidratos, insomnio global. Hipobulia.

Pasó al área COVID de emergencia pediátrica por resultado positivo a SARS-CoV-2, sin compromiso pulmonar. Resultados de laboratorio y de imágenes: Hb 12.8, leucocitos 7000, T4 libre 1,16 TSH:3,755, ácido fólico 7,58, Vit B12 604, marcadores tumorales negativos. IgM positivo para helicobacter pylori, marcadores tumorales negativos. Hisopado SARS-CoV-2 positivo. tomografía de abdomen y pelvis: Hígado con tamaño incrementado, con diámetro de lóbulo derecho de $143 \mathrm{~mm}$. Estómago parcialmente distendido, paredes se muestran levemente engrosada de aspecto inflamatorio. No signos de proceso neoproliferativo. Ausencia de lesiones focales. TAC Tórax: sin hallazgos significativos. Tac cerebral: Estudio tomográfico del encéfalo sin hallazgos significativos. Ecocardiograma dentro de límites normales. Escala Yale-Brown Obsessive Compulsive Scale (Y-BOCS) al ingreso de hospitalización, con un puntaje de 19, indicando manifestaciones clínica moderada de pensamientos obsesivos. 


\section{Diagnósticos multiaxial CIE-10:}

Eje I: Predominio de pensamientos o rumiaciones obsesivas (F42.0). Otros: Trastornos de la Ingestión de alimentos-TERIA(F50.8) Síndrome de Asperger(F84.5)

Eje II: Sin trastornos específicos del desarrollo psicológico

\section{Eje III: CI aparentemente normal}

Eje IV: Depleción de volumen (E86) Caquexia (R64) Dolor abdominal (R10.4) Desnutrición proteico calórica severa (E43) Dispepsia (K 30) D/C Reflujo gastro esofágico (K21.9) COVID-19, virus identificado (U07.1)

Eje V: Problemas relacionados con hechos estresantes que afecta a la familia (Z63.7)

Eje VI: $40 / 100$

Evaluado por pediatría quien realiza exámenes de laboratorio por la extrema baja ponderal y síntomas digestivos, descartando una causa infecciosa u oncológica. Obteniendo resultados séricos dentro de rangos normales. hallazgo de hepatomegalia en tomografía abdominal en relación con la desnutrición crónica. A la evaluación por nutrición: IMC 13,7. Score entre -2 y -3 Se inicia fase de estabilización según protocolo de desnutrición severa, monitoreo de glicemias, electrolitos. Recibe antibiótico empírico, vía enteral 80-100 $\mathrm{kcal}$ y volumen total $120 \mathrm{cc} / \mathrm{Kg} /$ día. Se inicia fórmula polimérica, tolerando vía oral de forma gradual. Asimismo, fue evaluado por oncología para descartar un proceso neoproliferativo, siendo los resultados negativos. Durante la evolución se torna nauseoso, sintomatología que va disminuyendo. En el cuarto día de hospitalización presenta peso de $26 \mathrm{Kg}$, talla $141.5 \mathrm{~cm}$, índice de masa corporal (IMC) $13.1 \mathrm{Sc} 0.95 \mathrm{H} 2 \mathrm{O}$ $1600 \mathrm{ml} / \mathrm{m} 2 /$ día. Tolera vía oral en forma gradual. A la evaluación por Psiquiatría del niño y del adolescente se obtiene datos previos de la infancia de TEA y TERIA, síntomas que han disminuido durante el tiempo, persistiendo en menor intensidad la restricción de algunos alimentos presentando una baja de peso que fue minimizado por familiares. Asimismo, en el examen psicopatológico predominan las ideas obsesivas relacionadas a la ingesta de comida por el temor a vomitar tornándose muy ansioso y con gran déficit funcional social, al inicio prefería dormir en posición sentado por el temor a vomitar, iniciando tratamiento con Sertralina $25 \mathrm{mg}$ /día, Olanzapina $2.5 \mathrm{mg} /$ día por sonda naso gástrica, dosis que al alta se ha ido aumentando: Sertralina $150 \mathrm{mg}$ / día y Olanzapina 10 mg/día. Presentando mejoría psicopatológica con relación al pensamiento obsesivo, disminución de síntomas ansiosos, y mejora en la funcionalidad, vuelve a clases escolares en forma virtual con muy buen rendimiento. Recibiendo psicoeducación el paciente y la familia, psicoterapia conductual individual, aún persistiendo restricción de ingesta de algunos alimentos y la psicoterapia familiar, la cual ha sido importante para la conciencia de trastorno mental y colaboración activa de madre en la recuperación de paciente.

\section{Discusión}

Trastorno de evitación y restricción de la ingesta de alimentos (TERIA) se da en pacientes con conductas alimentarias restrictivas o evitativas con una pérdida significativa de peso, compromiso del crecimiento, dependencia de suplementos nutricionales para satisfacer las necesidades energéticas diarias, deficiencia nutricional o afectación marcada con el funcionamiento psicosocial. Algunas de las características de la conducta alimentaria incluyen limitaciones en la variedad de alimentos ingeridos, falta de voluntad para probar nuevos alimentos conocida como neofobia alimentaria. Las tasas de TERIA oscilan entre el 5\% y el $14 \%$ entre los programas de urgencias pediátricas para pacientes hospitalizados y hasta el $22,5 \%$ en un programa de tratamiento diurno de urgencias pediátricas. En comparación con los pacientes con anorexia y bulimia nerviosa, los pacientes con TERIA son más jóvenes, con mayor prevalencia en varones y comúnmente se les diagnostica síntomas psiquiátricos y/o no psiquiátricos comórbidos ${ }^{(10)}$. Entre las comorbilidades no psiquiátricas son frecuentes los síntomas gastrointestinales y alergias alimentarias ${ }^{(11)}$.

Según los Centros para el Control y la Prevención de Enfermedades, 1 de cada 59 niños en Estados Unidos se le diagnostica un $\mathrm{TEA}^{(12)}$. En los pacientes con TEA, por algunas características sensoriales, como los comportamientos restringidos, hipersensibilidad e hiposensibilidad a algunos alimentos, es más frecuente presentarse la ingesta restringida, problemas de hábitos y patrones alimentarios que podría ocasionar una desnutrición crónica ${ }^{(13)}$. La selectividad alimentaria en el TEA afecta hasta un $95 \%$ de los niños con diagnóstico de autismo ${ }^{(14)}$.

En este artículo presentamos el caso de un paciente con diagnóstico del trastorno del espectro autista, por la afectación social, comunicación y los patrones restrictivos y repetitivos presentes desde la primera infancia, sin déficit intelectual y sin deterioro del lenguaje, con un grado de gravedad en la 
actualidad debajo del nivel 1, por no presentar interferencia en la comunicación social y que la inflexibilidad del comportamiento no dificulta su autonomía. En el CIE-10 cumple los criterios de síndrome de Asperger, diagnóstico que actualmente se considera dentro del trastorno del espectro autista en el DSM 5. Diagnóstico previo y de importancia con relación a la sintomatología del TOC como comorbilidad y que al completar la historia clínica del paciente permitió un abordaje integral, psicoterapia conductual y sobre todo en la psicoeducación a la madre y paciente para una mayor comprensión de sintomatología y mejor pronóstico.

Según algunos estudios, las personas con trastorno del espectro autista tienen dos veces más probabilidad de ser diagnosticadas con trastorno obsesivo compulsivo y las personas con trastorno obsesivo compulsivo tienen cuatro veces más probabilidad de un diagnóstico posterior con trastorno del espectro autista ${ }^{(15)}$. Con una tasa de comorbilidad del 6-37\% en la población pediátrica. En algunos estudios se ha encontrado síntomas más altos de TOC en madres de niños con TEA y el aumento de los síntomas de TEA en madres de niños con TOC. Las compulsiones del TOC son similares a las conductas restringidas, repetitivas y estereotipadas del TEA. Lo que podría diferenciarlas es la perturbación y ansiedad que presenta el paciente con TOC a diferencia de las conductas repetitivas del TEA son egosintónicas. La Escala Obsesiva Compulsiva de Yale-Brown para niños para el TEA(CYBOCSASD) se puede utilizar clínicamente para el diagnóstico y el seguimiento del tratamiento ${ }^{(16)}$.

Neurobiológicamente, tanto en el TOC y TEA se han encontrado alteraciones funcionales comunes en el córtex orbitofrontal, núcleo accumbens, cortex cingulado anterior (CCA) y caudado. Niveles de glutamato en el CCA están aumentados ${ }^{(17)}$. El contenido de rituales del TEA suele ser de repetición, orden y posición. Los rituales obsesivos más típicos del TOC son de contaminación y limpieza. Las obsesiones en el paciente con TEA y TOC suelen ser de contenido religiosas y presentar menos compulsiones de lavado y control ${ }^{(18,16)}$. En el tratamiento en niños y adolescente de comorbilidad del trastorno del espectro autista con síntomas del trastorno obsesivo compulsivo, tienen mayor evidencia de eficacia a la fluoxetina y la fluvoxamina. En el Trastorno obsesivo compulsivo, los inhibidores selectivos de la recaptación de serotonina (ISRS) tiene una efectividad del 60-70\%, la clomipramina es una opción también en niños a partir de los 10 años y un
$30 \%$ de los pacientes no responden al tratamiento. La combinación de ISRS y antipsicóticos puede mejorar los síntomas del TOC. Como parte del tratamiento, la colaboración de la familia es indispensable, así como la psicoeducación y la psicoterapia $^{(18)}$ Iniesta-Sepúlveda et al. (2018) realizaron un estudio con nueve adolescentes de 11 a 17 años, diagnosticados con TOC y TEA de alto funcionamiento, quienes recibieron terapia cognitiva conductual (TCC) que incorporaba la exposición con prevención de respuesta (ERP) entre 24-80 sesiones diarias. El $78 \%$ respondieron al tratamiento, con mejoras significativas en el deterioro funcional, la calidad de vida, la ansiedad y la depresión ${ }^{(19)}$.

Como parte de la investigación en el trastorno del espectro autista, hay evidencia que los niños con TEA tienen más probabilidad de presentar problemas relacionados con el tracto gastro intestinal con relación al microbiota intestinal, los sistemas inmunológicos, nervioso y la barrera mucosa. Una terapia potencial on los probióticos, dieto terapia y el trasplante de microbiota fecal (FMT), asimismo se enfatiza el importante papel del estrés oxidativo con relación a la disfunción mitocondrial, planteándose el uso de $\omega-3$, leucotrienos, ácido docosahexaenoico (DHA) y vitamina B12, utilizados como un nuevo enfoque en la terapia en los trastornos del espectro autista $^{(20)}$.

Entre las limitaciones que se encontraron en este caso clínico, es que, a pesar de las evidencias de asociación de trastornos alimentarios en niños con trastorno del espectro autista y las molestias gastrointestinales, se dejó de lado la intervención por gastroenterología durante la hospitalización como evaluación multidisciplinaria y parte importante para la recuperación del paciente, lo cual se está realizando el seguimiento por consultorio externo de nutrición y pediatría. Se requiere mayor investigación en el trastorno del espectro autista y sus comorbilidades a nivel nacional.

\section{Conclusión}

Es frecuente la asociación del trastorno del espectro autista (TEA) y trastorno obsesivo compulsivo, muchas veces siendo infradiagnosticado, lo cual interfiere en su intervención terapéutica temprana. La presencia de trastorno de conducta alimentaria es una forma característica del TEA por lo que es de importancia las comorbilidades y su repercusión en el pronóstico del TEA y la prevención de las complicaciones graves como en el caso que presentamos con una desnutrición proteico-calórica severa. 


\section{Aspectos físicos}

El paciente y sus familiares descrito en el reporte de caso brindaron su asentimiento para la publicación de su historial clínico, Contamos con el consentimiento del tutor del paciente.
Referencias bibliográficas

1. American Psychiatric Association-APA. Manual diagnóstico y estadístico de los trastornos mentales DSM-5 (5a. ed. --.?). Madrid: Editorial Médica Panamericana. 2014.

2. Suspes Cruz Y, Orejarena Serrano S. Trastorno de evitación y restricción de la ingestión de alimentos en Pediatría: un diagnóstico novedoso para una entidad frecuente en la práctica clínica. Pediatría integral 2020;2004(4):234.e1-234.e7

3. De Toro V, Aedo K, Urrejola P. Trastorno de Evitación y Restricción de la Ingesta de Alimentos (ARFID): Lo que el pediatra debe saber. Andes pediátrica.2021;92(2):298-307. https://dx.doi.org/10.32641/ andespediatr.v92i 2.2794

4.Plataforma digital única del Estado Peruano. $81 \%$ de personas tratadas por autismo en Perú son varones [Internet]. Lima; 2019 [citado el 1 de abril de 2019]. Disponible en: https://www.gob.pe/ institucion/minsa/noticias/27103-el-81-de-personas-tratadas-porautismo-en-peru-son-varones

5. Sadock BJ, M.D., Alcott Sadock V, M.D., Ruiz P, M.D. Sinopsis de Psiquiatría Ciencias del comportamiento/Psiquiatría Clínica. China: Wolters Kluwer. 2015.

6. Griffiths, DL, Farrell LJ, Waters AM, White SW. ASD Traits Among Youth with Obsessive-Compulsive Disorder. Child psychiatry and human development.2017;48(6):911-921. https://doi.org/10.10 07/s10578-017-0714-3

7. Tang B, Piazza CC, Dolezal D, Stein MT. Severe feeding disorder and malnutrition in 2 children with autism. Journal of developmental and behavioral pediatrics : JDBP.2011;32(3):264-267. https://doi.org/ 10.1097/DBP.0b013e3182138668

8. Martin AF, Jassi A, Cullen AE, Broadbent M, Downs J, Krebs G. Co-occurring obsessive-compulsive disorder and autism spectrum disorder in young people: prevalence, clinical characteristics and outcomes. European child \& adolescent psychiatry.2020; 29(11):1603-1611.https://doi.org/10.1007/s00787-020-01478-8

9. Flygare O, Andersson E, Ringberg H, Hellstadius AC, Edbacken J, Enander, J, Dahl M, Aspvall K, Windh I, Russell A, MataixCols, D, Rück C. Adapted cognitive behavior therapy for obsessivecompulsive disorder with co-occurring autism spectrum disorder: A clinical effectiveness study. Autism: the international journal of research and practice.2020;24(1):190-199. https://doi.org/ $10.1177 / 1362361319856974$

10. Norris ML, Spettigue WJ, Katzman DK. Update on eating disorders: current perspectives on avoidant/restrictive food intake disorder in children and youth. Neuropsychiatric disease and treatment.2016;12:213-218. https://doi.org/10.2147/NDT.S82538

11. Tsang KK, Hayes LC, Bujoreanu S, Samsel CB, Ibeziako PI.
Estudio de caracterización de pacientes que acudieron a un hospital pediátrico de cuidados agudos identificados con trastorno por evitación/restricción de la ingesta de alimentos. Pediatría hospitalaria. 2020;10 (7):600-607. DOI: 10.1542/ hpeds.2020-0010.

12. Crowley JG, Peterson KM, Fisher WW, Piazza CC. Treating food selectivity as resistance to change in children with autism spectrum disorder. Journal of applied behavior análisis.2020;53(4):2002-2023. https://doi.org/10.1002/jaba.711

13. Yanagimoto $\mathbf{Y}$, Ishizaki $\mathbf{Y}$, Kaneko K. Iron deficiency anemia, stunted growth, and developmental delay due to avoidant/restrictive food intake disorder by restricted eating in autism spectrum disorder. BioPsychoSocial medicine.2020; 14:8. https://doi.org/10.11 86/s13030-020-00182-y

14. Sharp WG, Berry RC, Burrell L., Scahill L, McElhanon BO. Scurvy as a Sequela of Avoidant-Restrictive Food Intake Disorder in Autism: A Systematic Review. Journal of developmental and behavioral pediatrics : JDBP. 2020;41(5):397-405. https://doi.org/ 10.1097/DBP.0000000000000782

15. Bedford SA, Hunsche MC, Kerns CM. Co-occurrence, Assessment and Treatment of Obsessive Compulsive Disorder in Children and Adults With Autism Spectrum Disorder. Current psychiatry reports.2020;22(10): 53. https://doi.org/10.1007/s11920-020-01176-

16. Pazuniak M, Pekrul SR. Obsessive-Compulsive Disorder in Autism Spectrum Disorder Across the Lifespan. Child and adolescent psychiatric clinics of North America 2020;29(2):419-432. https://doi.org/10.1016/j.chc.2019.12.003

17. Ortiz AE, Espelt C, Rosa M, Puig O, Lázaro L, Calvo R. Identificando compulsiones en niños y adolescentes con trastorno del espectro autista. Revista de Psiquiatría Infanto Juvenil. 2019;26(4): 24-40. DOI: 10.31766/revpsij.v36n4a4

18. Soutullo. Guía Esencial de Psicofarmacología del Niño y del Adolescente. Madrid: Editorial Médica Panamericana. 2016;16:221231.

19. Iniesta-Sepúlveda M. Nadeau JM, Ramos A, Kay B, Riemann BC, Storch EA. An Initial Case Series of Intensive Cognitive-Behavioral Therapy for Obsessive-Compulsive Disorder in Adolescents with Autism Spectrum Disorder. Child psychiatry and human development. 2018;49(1):9-19. https://doi.org/10.1107/s105 78-0170724-1

20.Hu T, Dong Y, He C, Zhao M, He Q. The Gut Microbiota and Oxidative Stress in Autism Spectrum Disorders (ASD). Oxidative medicine and cellular longevity.2020; 8396708. https://doi.org/ 10.11 $55 / 2020 / 8396708$

Contribución de autoría: María Antonieta Huete-Córdova, ha participado en la concepción del artículo, la recolección de datos, su redacción y aprobación de la versión final. Manuel David Arce-Martino ha colaborado en la redacción de datos y aprobación de versión final.

Conflicto de interés: Los autores no tienen conflicto de interés con la publicación de este trabajo.

Financiamiento: Autofinanciado.

Citar como: Huete-Córdova MA, Arce-Martino MD. Trastorno de evitación y restricción de la ingesta de alimentos (ARFID) con síntomas obsesivos en un niño con Trastorno del Espectro Autista, a propósito de un caso clínico. Diagnóstico(Lima). 2021;60(3):150-155.

DOI: 10.33734 /diagnostico.v60i3.304

Correspondencia: María Antonieta Huete Córdova. Hospital Nacional Guillermo Almenara Irigoyen -

Departamento de Psiquiatría. Av. Grau 800. Lima 1. Lima, Perú. 